\title{
Spectral Indices of Stars at Super-solar Regime
}

\author{
Alberto Buzzoni \\ Telescopio Nazionale Galileo, A.P. 565, 38700 Santa Cruz de La Palma (Tf), \\ Spain, \\ and Osservatorio Astronomico di Brera, Milano Italy \\ Miguel Chavez \\ Instituto Nacional de Astrofísica, Optica y Electrónica, A.P. 51 y 216, 72000 \\ Puebla, Mexico \\ Maria Lucia Malagnini \\ Dipartimento di Astronomia, Università degli Studi di Trieste, Via G.B. Tiepolo \\ 11, 34131 Trieste, Italy \\ Carlo Morossi \\ Osservatorio Astronomico di Trieste, Via G.B. Tiepolo 11, 34131 Trieste, Italy
}

\begin{abstract}
We derived Lick narrow-band indices for 139 candidate super metal-rich stars of different luminosity class for a sample of which fundamental atmosphere parameters have been obtained from a grid of synthetic reference spectra by Malagnini et al. (2000). Indices include Iron Fe50, Fe52, Fe53, and Magnesium Mgb and $\mathrm{Mg}_{2}$ features. By comparing models and observations, no evidence is found for nonstandard $\mathrm{Mg}$ vs. Fe relative abundance (i.e. $[\mathrm{Mg} / \mathrm{Fe}]=0$, on the average, for our sample). Both the Worthey et al. (1994) and Buzzoni et al. (1992, 1994) fitting functions are found to suitably match the data, and can therefore confidently be extended for population synthesis applications also to super-solar metallicity regimes.
\end{abstract}

Keywords: stars: fundamental parameters, galaxy: stellar content

\section{The Databases}

As a useful tool to investigate the distinctive properties of stellar aggregates, the fitting-function technique (Buzzoni 1995; Worthey et al. 1995) has been extensively used in population synthesis models to reproduce integrated spectroscopic indices, even in super metal-rich (SMR) systems such as elliptical galaxies or bulges of spirals.

Since these relations mainly derive from a fit of the local stellar population, with $Z \sim Z_{\odot}$, it is important to confidently assess their validity also at super-solar metallicity regimes. For this reason, we derived Lick narrow-band indices from spectroscopic observations of a sample of $139 \mathrm{SMR}$ stars with $[\mathrm{Fe} / \mathrm{H}] \geq+0.1$, comparing with a suitable theoretical database of synthetic reference spectra. A summary

\footnotetext{
* Based on observations collected at the INAOE "G. Haro" Observatory, Cananea (Mexico).
} 
Table I. The Databases.

\begin{tabular}{ll}
\hline Observations & Synthetic Spectra \\
\hline 139 Pop I Stars & Chavez et al. 1997 collection \\
300 spectra & 693 spectra \\
$4600 \div 5500 \AA$ & $4850 \div 5400 \AA$ \\
$R=\Delta \lambda / \lambda=2000$ & $R=\Delta \lambda / \lambda=250000$ \\
{$[F e / H] \geq 0.1$} & {$[M / H]=-1.0 \rightarrow+0.5$} \\
$\mathrm{M} \rightarrow$ F spectral type & $4000 K<T_{\text {eff }}<8000 \mathrm{~K}$ \\
$\mathrm{I} \rightarrow$ V MK luminosity class & $\log \mathrm{g}=1.0 \rightarrow 5.0$ \\
\hline
\end{tabular}

of the main characteristics of the databases is given in Table 1 (see also Chavez et al. 1997, and Malagnini et al. 2000 for further details).

From the observed spectra, Iron Fe50, Fe52, and Fe53, as well as Magnesium $\mathrm{Mgb}$ and $\mathrm{Mg}_{2}$ indices have been computed according to Worthey et al. (1994, hereafter W94). Calibration to the standard system has been done by using the subset of 49 stars in common with the Lick original database, and/or with Buzzoni et al. (1992, 1994; B92 and B94, respectively).

\section{Index residuals and atmosphere parameters}

The $(\mathrm{O}-\mathrm{C})$ index residuals obtained by comparing observations with the corresponding fitting spectra from the theoretical database have been analyzed vs. atmosphere fiducial parameters, according to Malagnini et al. (2000). Figure 1 shows the residual distribution for $\mathrm{Mg}_{2}$ and the combined Iron index $<\mathrm{Fe}\rangle=(F e 52+F e 53) / 2$ vs. $[\mathrm{Fe} / \mathrm{H}]$. Solid dots mark the "fair" subsample of 73 stars with more confident fundamental parameters (cf. Malagnini et al. 2000).

The four points with the largest (negative) residuals correspond to the coolest stars in our sample $\left(T_{\text {eff }} \leq 4000 \mathrm{~K}\right)$, and reflect the partial inadequacy of the theoretical database at this temperature range. As far as the remaining sample of 135 stars is considered, however, the residual distribution for all indices is consistent with a zero-average hypothesis. Quite remarkably, we also verified the lack of any negative correlation between $\mathrm{Mg}$ and $\mathrm{Fe}(\mathrm{O}-\mathrm{C})$ residuals. This indicates that a non-solar partition for the $[\mathrm{Mg} / \mathrm{Fe}]$ relative abundance can confidently be ruled out for our stars. 


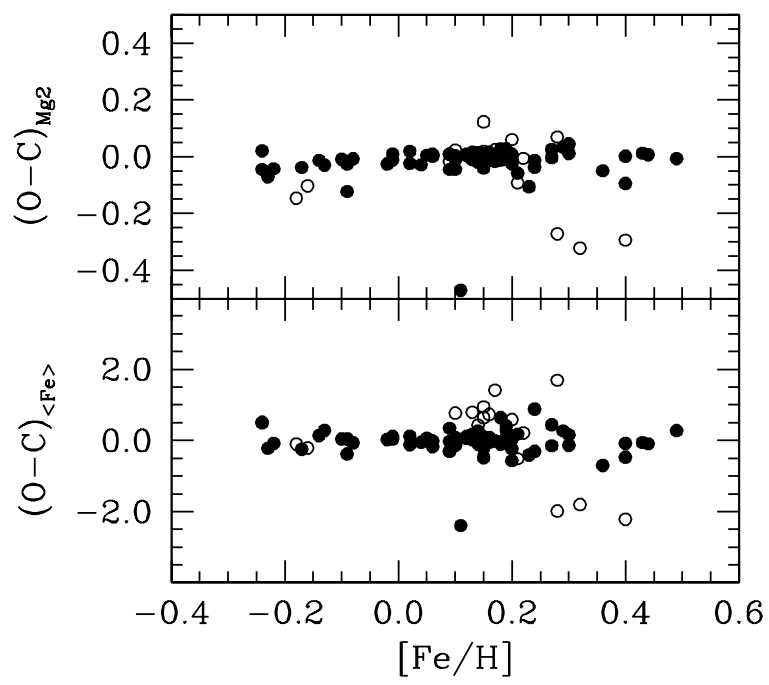

Figure 1. (O-C) index residuals vs. $[\mathrm{Fe} / \mathrm{H}]$. The 73 stars in the "fair" subsample, with more confident atmosphere parameters, are singled out ("•" markers). Excluding the four outliers (the coolest stars in our sample with $T_{\text {eff }} \leq 4000 \mathrm{~K}$ ) $(\mathrm{O}-\mathrm{C})$ residuals are consistent with a zero average.

\section{Fitting Functions}

Analytical fitting functions, giving the $\mathrm{Fe}$ and $\mathrm{Mg}$ index strength vs. stellar atmosphere parameters across the H-R diagram, have been provided by B92, B94, and W94. To check self-consistency of these functions also in the SMR regime we studied their index residuals with respect to our observations. Figure 2 summarizes the results for $\mathrm{Mg}_{2}$ and $<\mathrm{Fe}>$.

The $(\mathrm{O}-\mathrm{C})$ distribution in both panels is consistent with a zero average residual confirming that the two sets of equations properly account for high-metallicity stars, at least in the range of the atmosphere parameters sampled by our stars. The W94 fitting function is slightly more accurate than the B92 one (points spread for the "fair" sample is $\sigma\left(\mathrm{Mg}_{2}\right)= \pm 0.047$ mag vs. \pm 0.052 mag for the latter case), but at cost of a more elaborated multi-branch analytical fit.

A similar behaviour is found for the $<\mathrm{Fe}>$ index, with $\sigma(<\mathrm{Fe}>$ )$= \pm 0.61 \AA$ and $\pm 0.34 \AA$ with respect to $\mathrm{B} 94$ and W94, respectively. We verified that the skewed positive residuals with respect to B94, evident from the figure, mostly come from the warmer stars in the sample $\left(T_{\text {eff }} \gtrsim 6700 \mathrm{~K}\right)$ for which the B94 output predicts a vanishing Fe52 index. 

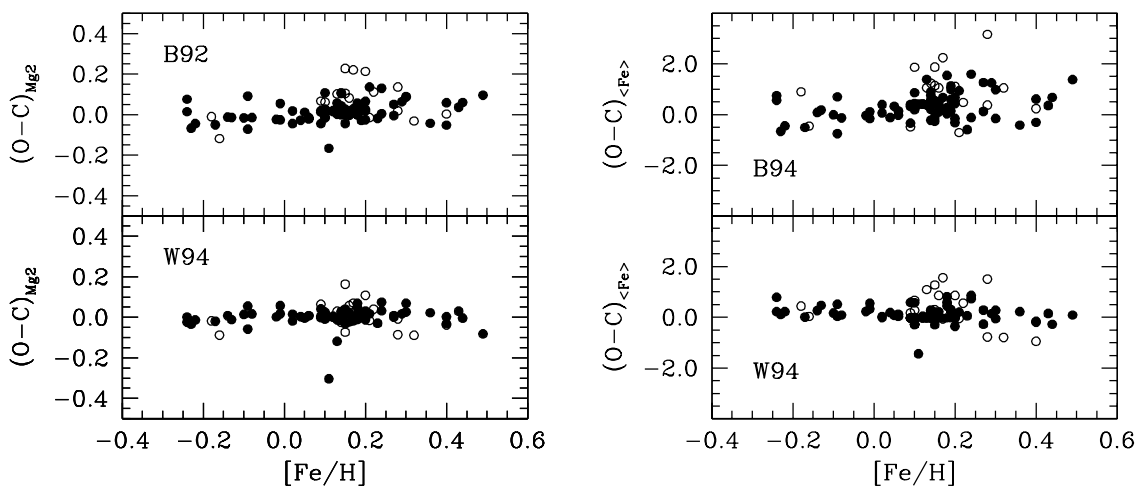

Figure 2. The $\mathrm{Mg}_{2}$ and $<\mathrm{Fe}>$ index residuals (in the sense [Observed - Computed]) with respect to the Buzzoni et al. $(1994,1994)$ and Worthey et al. (1994) fitting functions are displayed vs. metallicity. Like in Fig. 1, solid dots mark stars in the "fair" subsample. Both $(\mathrm{O}-\mathrm{C})$ distributions are consistent with a zero average.

\section{Acknowledgements}

This work received partial financial support from the Italian MURST via COFIN '98/'00, and 60\% grants, and from the Mexican CONACyT via grant 28506 -E.

\section{References}

Buzzoni, A. Spectrophotometric Properties of Elliptical Galaxy Cores. In A. Buzzoni, A. Renzini and A. Serrano editors, Fresh Views of Elliptical Galaxies. ASP San Francisco, p. 189, 1995

Buzzoni, A., Gariboldi, G. and Mantegazza, L. The Magnesium $\mathrm{Mg}_{2}$ Index as an Indicator of Metallicity in Elliptical Galaxies. AJ, 103:1814-1827, 1992. [B92]

Buzzoni, A., Mantegazza, L. and Gariboldi, G. Metal Enrichment in Elliptical Galaxies and Globular Clusters through the Study of Iron and $\mathrm{H} \beta$ Spectral Indices. AJ, 107:513-529, 1994. [B94]

Chavez, M., Malagnini, M. L. and Morossi, C. An Atlas of High Resolution Synthetic Spectra in the Wavelength Region 4850-5400 A. A\&ApS, 126:267-272, 1997.

Malagnini, M. L., Morossi, C., Buzzoni, A. and Chavez, M. Observations and Atmospheric Parameters of Super Metal-rich Candidates. PASP, 112:1455-1466, 2000.

Worthey, G., Faber, S.M., Gonzalez, J.J., Burstein, D. Old Stellar Populations. V: Absorption Feature Indices for the Complete LICK/IDS Sample of Stars. ApJS, 94:687-722, 1994. [W94]

Worthey, G., Trager, S. C. and Faber, S. M. The Galaxian Age-Metallicity Relation. In A. Buzzoni, A. Renzini and A. Serrano editors, Fresh Views of Elliptical Galaxies. ASP San Francisco, p. 203, 1995 